Pesq. Vet. Bras. 35(7):643-648, julho 2015 DOI: $10.1590 / \mathrm{S} 0100-736 \mathrm{X} 2015000700007$

\title{
Resistência anti-helmíntica em rebanhos caprinos nos biomas Caatinga e Mata Atlântica ${ }^{1}$
}

\author{
Simone L. Borges ${ }^{2 *}$, Alex A. Oliveira², Lívia R. Mendonça², Sabrina M. Lambert², \\ Juliana M. Viana ${ }^{2}$, Sandra M. Nishi ${ }^{2}$, Fred da Silva Julião ${ }^{3}$ e Maria Angela O. Almeida ${ }^{2}$
}

\begin{abstract}
Borges S.L., Oliveira A.A., Mendonça L.R., Lambert S.M., Viana J.M., Nishi S.M., Julião F.S. \& Almeida M.A.O. 2015. [Anthelmintic resistance in goat herds in the Caatinga and Mata Atlântica biomes.] Resistência anti-helmíntica em rebanhos caprinos nos biomas Caatinga e Mata Atlântica. Pesquisa Veterinária Brasileira 35(7):643-648. Escola de Medicina Veterinária e Zootecnia, Universidade Federal da Bahia, Av. Ademar de Barros 500, Salvador, BA 40170-110, Brazil. E-mail: simlobor@yahoo.com.br

The use of anthelmintic drugs for long periods as the main measure control of gastrointestinal nematodes (GINs) has led to the inefficacy of levamisole, benzimidazoles and macrocyclic lactones. This study describes the in vivo anthelmintic activity against natural trichostrongyle nematodes populations in goats. We selected 18 herds from the Caatinga $(n=12)$ and Mata Atlântica ( $n=6)$ biomes, Bahia State, Brazil, raised in communal pastures in semiarid region. Groups of 8 to 10 goats were treated with albendazole (ABZ), ivermectin (IVM), levamisole (LEV), moxidectin (MOX), and closantel (CLOS). The results of the Fecal Egg Count Reduction Test indicated simultaneous resistance of Haemonchus sp. and Trichostrongylus spp. genera against albendazole (ABZ), ivermectin (IVM), levamisole (LEV), moxidectin (MOX), and closantel (CLOS). The efficacy percentages ranged from 0 to $92 \%, 0$ to $75 \%, 0$ to $91 \%, 69$ to $97 \%$, and 0 to $85 \%$ for ABZ, IVM, LEV, MXD and CLOS respectively in the Caatinga bioma, and 0 to $59 \%$ for ABZ and 9 to 59\% for IVM in the Mata Atlântica biome. Most herds showed efficacy lower than $95 \%$ for anthelmintics, with the exception of one herd in which the efficacy for MOX was $97 \%$. The results indicated the presence of GINs resistant to main anthelmintics classes in goat herds in these biomes.
\end{abstract}

INDEX TERMS: Anthelmintic resistance, nematodes, benzimidazole, avermectin, imidozothiazole, goats, ruminants.

RESUMO.- A utilização de anti-helmínticos por longos períodos como principal medida de controle das parasitoses gastrintestinais de ruminantes levou a ineficácia aos levamisol, benzimidazóis e avermectinas. Este estudo descreve a atividade anti-helmíntica in vivo em populações naturais de nematoides trichostrongilídeos de caprinos. Foram selecionados 18 rebanhos provenientes dos biomas Caatinga $(n=12)$ e Mata Atlântica ( $n=6)$, do Estado da Bahia, Brasil, criados em pastagens comunais em região semiárida. Gru-

\footnotetext{
${ }^{1}$ Recebido em 25 de novembro de 2014.

Aceito para publicação em 25 de maio de 2015.

${ }^{2}$ Escola de Medicina Veterinária e Zootecnia, Universidade Federal da Bahia (UFBA), Av. Ademar de Barros 500, Salvador, BA 40170-110, Brasil. *Autor para correspondência: simlobor@yahoo.com.br

${ }^{3}$ Instituto Federal de Educação, Ciência e Tecnologia Baiano, Campus Santa Inês, BR-420 (Rodovia Santa Inês-Ubaíra), Zona Rural, BA 45320000, Brasil.
}

pos de oito a 10 animais foram tratados com albendazol (ABZ), ivermectina (IVM), levamisol (LEV), moxidectina (MOX) e closantel (CLOS). Os resultados do Teste de Redução da Contagem de Ovos nas Fezes indicaram resistência simultânea dos gêneros Haemonchus sp. e Trichostrongylus spp. para o ABZ, IVM, LEV, MOX e CLOS. As percentagens de eficácia variaram de 0-92\%, 0-75\%, 0-91\%, 69-97\% e 0-85\% para o ABZ, IVM, LEV, MXD e CLOS, respectivamente, no bioma Caatinga e $0-59 \%$ para o ABZ e 9-59\% para o IVM no bioma Mata Atlântica. Verificou-se nos rebanhos eficácia inferior a 95\% para estes anti-helmínticos, com exceção de um único rebanho no qual a eficácia para MOX foi de 97\%, o que sugere a presença de NGIs resistentes aos principais classes de anti-helmínticos em rebanhos caprinos destes biomas.

TERMOS DE INDEXAÇÃO: Resistência anti-helmíntica, nematoides, benzimidazol, avermectina, imidozotiazol, caprinos, ruminantes. 


\section{INTRODUÇÃO}

A complexidade do controle de nematoides gastrintestinais (NGIs) em pequenos ruminantes vem aumentando em consequência da resistência aos anti-helmínticos.

A resistência ocorre pela exposição dos NGIs ao anti-helmíntico, como resultado da habilidade de uma população destes parasitos de sobreviver ao efeito das doses terapêuticas em relação à população suscetível da mesma espécie (Prichard et al. 1980). Os fatores que influenciam a seleção destas populações resistentes incluem o uso indiscriminado e excessivo de anti-helmínticos, a frequente circulação de animais entre rebanhos e a inexistência de procedimentos de quarentena para os recém-chegados (Howell et al. 2008), fatores estes fortemente associados ao tipo de criação de pequenos ruminantes em sistema de pecuária familiar no semiárido brasileiro.

No nordeste do Brasil a resistência de nematoides aos imidozotiazóis, benzimidazóis e lactonas macrociclícas é cada vez mais comprovada (Vieira \& Cavalcante 1999, Ahid et al. 2007, Coelho et al. 2010) e a ascensão do índice de endemismo de nematoides resistentes tem modificado a dinâmica da produção de pequenos ruminantes, em decorrência de maior parasitismo dos animais e a ineficiência do controle antiparasitário.

A resistência anti-helmíntica em nematoides gastrintestinais de caprinos, ao contrário dos ovinos, ainda não é bem documentada. Mundialmente, poucos estudos foram realizados com caprinos criados em condições semiáridas, e alguns destes foram desenvolvidos no nordeste brasileiro (Rodrigues et al. 2007, Melo et al. 2009, Lima et al. 2010a, Lima et al. 2010b) e na África (Waruiru et al. 1998, Kumsa \& Abebe 2009).

A criação de caprinos por pequenos e médios produtores, nos biomas Caatinga (município de Cansanção) e Mata Atlântica (Vale do Jiquiriçá) no território baiano, ocorre em sistema de manejo extensivo e comunal, com predomínio muitas vezes do modelo produtivo conhecido como "fundo de pasto", que é caracterizado pela criação de caprinos e ovinos em áreas desprovidas de cerca, de modo que os animais de diversos criadores utilizam áreas coletivas de vegetação nativa (Voltolini et al. 2011). Por certo, o pastoreio em pastagens compartilhadas favorece o fluxo gênico entre populações de parasitos, o que pode refletir na variação da resposta dos nematoides aos tratamentos anti-helmínticos (Silvestre et al. 2002).

Considerando-se que para um controle parasitário eficaz é necessário o emprego de anti-helmínticos, o conhecimento do nível de resistência de populações de nematoides permitirá o ajuste de práticas de manejo, a fim de mitigar os seus efeitos no controle de helmintos. Assim sendo, um estudo a campo utilizando o Teste de Redução da Contagem de Ovos nas Fezes (TRCOF) foi realizado, para verificar a ocorrência de resistência parasitária em rebanhos caprinos dos biomas Caatinga e Mata Atlântica no território baiano.

\section{MATERIAL E MÉTODOS}

No bioma Caatinga, o estudo ocorreu de março de 2011 a julho de 2012, no município de Cansanção (1040’02" S e 39²9' 57" W), nordeste da Bahia, que apresenta tipos climáticos semiárido e árido, médias anuais de temperatura e precipitação pluviométrica de $23,6^{\circ} \mathrm{C}$ e $477 \mathrm{~mm}$, respectivamente e período chuvoso de março a maio. A vegetação nativa é caracterizada por Caatinga arbórea aberta com palmeiras e Caatinga arbórea densa sem palmeiras. No bioma Mata Atlântica, os testes foram realizadas, entre janeiro e junho de 2013, nos municípios de Santa Inês, Cravolândia e

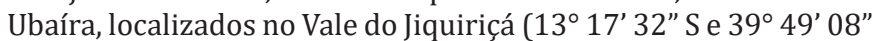
W), sudoeste baiano, cujos os tipos climáticos variam de úmido e subúmido a seco, com médias anuais de temperatura entre 18,8 e $25,4^{\circ} \mathrm{C}$ e de precipitação pluviométrica de 800 a $2000 \mathrm{~mm}$ e período chuvoso de março a maio. A vegetação nativa é caracterizada por Floresta Estacional Decidual e Semidecidual (Bahia 2013).

O levantamento incluiu 18 rebanhos caprinos, de propriedades de cunho familiar, com finalidade principal de produção de carne. Doze rebanhos encontravam-se em diferentes áreas no município de Cansanção: Alto Lindo (Rebanho 1), Assentamento Morada Nova (Rebanho 2), Assentamento Nova Vida (Rebanhos 3 a 9) e Assentamento Belo Monte (Rebanhos 10 a 12) e outros seis situados nos municípios de Santa Inês (Rebanhos 1 a 3), Cravolândia (Rebanhos 4 e 5) e Ubaíra (Rebanho 6). Para identificação do perfil do sistema de produção foram realizadas entrevistas com os criadores. Nos rebanhos no município Cansanção, caprinos, ovinos e bovinos eram criados, predominantemente, no sistema de produção de "fundo de pasto". Os rebanhos caprinos do Vale do Jiquiriçá eram mantidos em regime extensivo tradicional e alguns deles compartilhavam o pasto com poucos bovinos. Em ambos os rebanhos ocorria a pernoite dos animais no aprisco. 0 número de caprinos nestes criatórios variou de 40 a 150 .

Os caprinos sem raça definida, de seis a 18 meses de idade, em estado de higidez adequado, se apresentavam naturalmente infectados por nematoides gastrintestinais, pois não foram tratados com anti-helmíntico por no mínimo 60 dias antes do teste, como preconizado por Coles et al. (1992).

Para o Teste de Redução da Contagem de Ovos nas Fezes (TRCOF) (Coles et al. 1992) foram incluídos apenas os animais com contagem de ovos por grama de fezes (OPG) superior a 200, e empregou-se no tratamento o albendazol (ABZ) $(5 \mathrm{mg} / \mathrm{kg}$ de peso vivo, via oral - Aldazol ${ }^{\circledR} /$ Vallée); ivermectina (IVM) $(0,2 \mathrm{mg} / \mathrm{kg}$, via oral - Ivomec ovino ${ }^{\circledR} /$ Merial); levamisol (LEV) (5mg/kg, via oral - Ripercol ${ }^{\circledR} /$ Fort Dodge); moxidectina (MOX) $(0,2 \mathrm{mg} / \mathrm{kg}$, via oral - Cydectin/Fort Dodge) e closantel (CLOS) (10mg/kg, via oral - Diantel $\left.{ }^{\circledR} / \mathrm{Irfa}\right)$. Como nas formulações com LEV e MOX não havia indicação para tratamento em caprinos, usou-se a dose recomendada para ovinos.

Os grupos foram compostos por oito a 10 caprinos, na dependência do número de animais por rebanho, conforme metodologia recomendada por Molento et al. (2011), que adota o número mínimo de oito animais por cada grupo. Os 12 rebanhos do bioma Caatinga foram avaliados para o ABZ, LEV e IVM, utilizando 10 animais por produto $(10$ caprinos $\times 3$ produtos $\times 12$ rebanhos $=$ 360 animais). Apenas nos rebanhos 10, 11 e 12 deste bioma foram testados a MOX e o CLOS, sendo os grupos composto por nove animais ( 9 caprinos $\times 2$ produtos $\times 3$ rebanhos $=54$ animais). No bioma Mata Atlântica, pelo número limitado de animais nos rebanhos, foram testados exclusivamente o ABZ e a IVM nos seis rebanhos. Os grupos foram formados com nove animais ( 9 caprinos $\mathrm{x}$ 2 produtos x 6 rebanhos $=108$ animais). Em cada um dos 18 rebanhos estudados foi incluindo um grupo controle, formado por oito ou 10 animais, que não receberam qualquer medicamento.

As amostras de fezes foram coletadas de cada animal no dia do tratamento e 14 dias após, sendo processadas para contagem de ovos (OPG) e de larvas de $3^{\circ}$ estádio obtidas de coproculturas (Ueno \& Goncalves 1998). A população de nematoides foi considerada resistente quando a redução da contagem de ovos após o tratamento foi inferior ou igual a $90 \%$ e o intervalo de confiança a 95\% inferior a 90\% (Coles et al. 1992). 
Os resultados do OPG e do percentual de larvas obtidos no TRCOF foram analisados pelo programa RESO 2.01 (CSIRO) para determinar a eficácia dos anti-helmínticos. A comparação entre os valores do OPG e eficácia de ABZ e IVM entre os biomas Caatinga e Mata Atlântica foi realizada pelo teste não paramétrico Mann-Whitney ( $\mathrm{p} \leq 0,05)$, por meio do programa SPSS v. 17.0 (IBM, EUA).

\section{RESULTADOS}

\section{Bioma Caatinga}

$\mathrm{Na}$ análise do questionário respondido pelos produtores, verificou-se que a desparasitação ocorreram a cada três $(38 \%)$ ou quatro $(26 \%)$ vezes ao ano, realizadas principalmente no período seco (38\%) ou logo após as primeiras chuvas (35\%). Em $77 \%$ dos rebanhos o vermífugo é aplicado a todos os animais numa mesma ocasião e a dosagem é calculada pela estimativa visual do peso. Também se constatou o uso mais frequente das avermectinas $(66,6 \%)$, seguido dos benzimidazóis $(44,4 \%)$ e imidozotiazóis $(33,3 \%)$, sendo a troca do anti-helmíntico realizada a cada vermifugação $(29 \%)$ ou semestralmente $(20 \%)$.

A resistência múltipla anti-helmíntica foi demonstrada em rebanhos caprinos do município de Cansanção (Quadro 1). Os valores do OPG variaram de 560 a 1455 no grupo controle, com média de 897,4. Não houve diferença estatística entre as médias de OPG dos animais dos grupos controle e tratado. A eficácia variou de $0-92 \%$ para o ABZ, de 0-75\% para IVM e de $0-91 \%$ para LEV.

A MOX e o CLOS foram avaliados nos rebanhos 10, 11 e 12, em virtude da ineficácia dos demais anti-helmínticos testados. A eficácia da MOX foi $69 \%, 80 \%$ e $97 \%$ e do CLOS de $0 \%, 63 \%$ e $85 \%$ para os Rebanhos 10,11 e 12 respectivamente.

A eficácia foi baixa para o gênero Haemonchus. Apenas nos rebanhos 1 e 2 a eficácia foi de $98 \%$ para ABZ e $95 \%$ para IVM (Quadro 2). Eficácia mínima, abaixo de $7 \%$ foi notada para o LEV. Diferentemente para o gênero Trichostrongylus, a eficácia do LEV foi igual ou maior a 95\% em $60 \%$ dos rebanhos. A ocorrência de resistência em populações de Trichostrongylus spp. foi também conferida a outras duas bases químicas. Em 33,3\% (4/12) e 18,2\% (2/12)

Quadro 1. Médias da contagem de ovos (OPG) de nematoides gastrintestinais em fezes de caprinos do bioma Caatinga e eficácia dos anti-helmínticos albendazol, ivermectina e levamisol

\begin{tabular}{|c|c|c|c|c|c|c|c|}
\hline \multirow[t]{2}{*}{ Rebanhos } & \multirow{2}{*}{$\begin{array}{c}\text { OPG } \\
\text { Controle } \\
\text { negativo }\end{array}$} & \multicolumn{2}{|c|}{ Albendazol } & \multicolumn{2}{|c|}{ Ivermectina } & \multicolumn{2}{|c|}{ Levamisol } \\
\hline & & $\mathrm{OPG}$ & $\begin{array}{c}\text { Eficácia } \\
(\%)\end{array}$ & $\mathrm{OPG}$ & $\begin{array}{c}\text { Eficácia } \\
(\%)\end{array}$ & $\mathrm{OPG}$ & $\begin{array}{c}\text { Eficácia } \\
(\%)\end{array}$ \\
\hline 1 & 1411 & 664 & 53 & 968 & 31 & - & - \\
\hline 2 & 815 & 517 & 37 & 400 & 51 & - & - \\
\hline 3 & 685 & 461 & 33 & 360 & 47 & 61 & 91 \\
\hline 4 & 831 & 750 & 0 & 1219 & 0 & 286 & 66 \\
\hline 5 & 785 & 67 & 92 & 195 & 75 & 312 & 60 \\
\hline 6 & 830 & 842 & 0 & 619 & 25 & 2233 & 0 \\
\hline 7 & 1320 & 1028 & 22 & 737 & 44 & 422 & 68 \\
\hline 8 & 1455 & 1100 & 24 & 2185 & 0 & 600 & 59 \\
\hline 9 & 595 & 1413 & 0 & 1046 & 0 & 450 & 24 \\
\hline 10 & 851 & 317 & 63 & 730 & 14 & 747 & 12 \\
\hline 11 & 631 & 356 & 44 & 444 & 30 & 143 & 77 \\
\hline 12 & 560 & 389 & 31 & 345 & 38 & 560 & 89 \\
\hline Média & 897,4 & 658,7 & 33,3 & 770,7 & 29,6 & 581,4 & 54,6 \\
\hline
\end{tabular}

Quadro 2. Eficácia (\%) dos princípios ativos albendazol (ABZ), levamisol (LEV) e ivermectina (IVM) para os gêneros Haemonchus spp. e Trichostrongylus spp. de caprinos do bioma Caatinga

\begin{tabular}{|c|c|c|c|c|c|c|c|c|}
\hline \multirow{2}{*}{ Rebanhos } & \multicolumn{4}{|c|}{ Haemonchus spp. } & \multicolumn{4}{|c|}{ Trichostrongylus spp. } \\
\hline & $\begin{array}{c}\text { Frequência } \\
\text { de larvas } \\
\text { no controle } \\
\text { negativo }\end{array}$ & ABZ & LEV & IVM & $\begin{array}{c}\text { Frequência } \\
\text { de larvas } \\
\text { no controle } \\
\text { negativo }\end{array}$ & ABZ & LEV & IVM \\
\hline 1 & 85 & 98 & - & 65 & 15 & 2 & - & 1 \\
\hline 2 & 79 & 61 & - & 95 & 21 & 39 & - & 5 \\
\hline 3 & 75 & 32 & 0 & 1 & 25 & 68 & 100 & 99 \\
\hline 4 & 81 & 16 & 6 & 0 & 19 & 84 & 94 & 100 \\
\hline 5 & 72 & 29 & 2 & 6 & 28 & 71 & 98 & 94 \\
\hline 6 & 76 & 30 & 5 & 28 & 21 & 70 & 95 & 72 \\
\hline 7 & 69 & 4 & 7 & 53 & 31 & 96 & 93 & 47 \\
\hline 8 & 56 & 10 & 0 & 6 & 44 & 90 & 26 & 74 \\
\hline 9 & 83 & 1 & 0 & - & 17 & 99 & 100 & - \\
\hline 10 & 13 & 0 & 5 & 8 & 87 & 100 & 93 & 92 \\
\hline 11 & 0 & 30 & 2 & 51 & 100 & 70 & 98 & 49 \\
\hline 12 & 0 & 4 & 3 & 43 & 100 & 96 & 97 & 57 \\
\hline Média & 57,4 & 31,9 & 3,0 & 32,4 & 42,3 & 68,1 & 89,4 & 62,7 \\
\hline
\end{tabular}

Quadro 3. Médias da contagem de ovos (OPG) de nematoides gastrintestinais em fezes de caprinos do bioma Mata Atlântica e eficácia dos anti-helmínticos albendazol e ivermectina

\begin{tabular}{|c|c|c|c|c|c|}
\hline \multirow[t]{2}{*}{ Rebanhos } & \multirow{2}{*}{$\begin{array}{c}\text { OPG } \\
\text { Controle } \\
\text { negativo }\end{array}$} & \multicolumn{2}{|c|}{ Albendazol } & \multicolumn{2}{|c|}{ Ivermectina } \\
\hline & & $\mathrm{OPG}$ & $\begin{array}{c}\text { Eficácia } \\
(\%)\end{array}$ & $\mathrm{OPG}$ & $\begin{array}{c}\text { Eficácia } \\
(\%)\end{array}$ \\
\hline 1 & 717 & 805 & 0 & 605 & 16 \\
\hline 2 & 975 & 733 & 25 & 640 & 34 \\
\hline 3 & 1665 & 3140 & 0 & 1355 & 19 \\
\hline 4 & 1786 & 733 & 59 & 736 & 59 \\
\hline 5 & 1186 & 700 & 41 & 620 & 48 \\
\hline 6 & 1261 & 1864 & 0 & 1150 & 9 \\
\hline Média & 1265,0 & 1329,2 & 20,8 & 851,0 & 30,8 \\
\hline
\end{tabular}

dos rebanhos a eficácia do ABZ e IVM, respectivamente, foi igual ou superior a 95\%. Poucas larvas do gênero Oesophagostomum spp. foram observadas nas coproculturas e por este motivo não se analisou a eficácia.

\section{Bioma Mata Atlântica}

Sobre a criação de caprinos no Vale do Jiquiriçá verificou-se que a frequência anual de desparasitação variou de duas $(40 \%)$ a $12(40 \%)$. Em 83,3\% dos rebanhos o vermífugo é administrado a todos os animais numa mesma ocasião, e para o cálculo da dosagem do medicamento o peso dos animais é estimado visualmente, conforme citado por $100 \%$ dos criadores. Os benzimidazóis (67\%) foram os mais usados no tratamento dos caprinos e em frequência semelhante às avermectinas (16,5\%) e imidozotiazóis $(16,5 \%)$. A maioria dos criadores $(60 \%)$ informou que não tinham critérios para a troca do anti-helmíntico.

A resistência anti-helmíntica foi igualmente demonstrada em rebanhos caprinos deste bioma. O OPG variou de 717 a 1786 no grupo controle, sendo a média de 1265,0 (Quadro 3 ), contudo não houve diferença estatística entre as médias de OPG dos animais dos grupos controle e tratado. A eficácia variou de $0 \%$ a $59 \%$ para o ABZ e de $9 \%$ a $59 \%$ para IVM.

As frequências de larvas dos gêneros Haemonchus e Trichostrongylus variaram, respectivamente, de $29 \%$ a $63 \%$ e 
Quadro 4. Eficácia (\%) dos princípios ativos albendazol (ABZ) e ivermectina (IVM) para os gêneros Haemonchus spp. e Trichostrongylus spp. de caprinos do bioma Mata Atlântica

\begin{tabular}{|c|c|c|c|c|c|c|}
\hline \multirow[t]{2}{*}{ Rebanhos } & \multicolumn{3}{|c|}{ Haemonchus spp. } & \multicolumn{3}{|c|}{ Trichostrongylus spp. } \\
\hline & $\begin{array}{c}\text { Frequência } \\
\text { de larvas no } \\
\text { controle negativo }\end{array}$ & $\mathrm{ABZ}$ & IVM & $\begin{array}{c}\text { Frequência } \\
\text { de larvas no } \\
\text { controle negativo }\end{array}$ & $\mathrm{ABZ}$ & IVM \\
\hline 1 & 37 & 0 & 0 & 63 & 8 & 26 \\
\hline 2 & 29 & 70 & 0 & 69 & 12 & 70 \\
\hline 3 & 46 & 0 & 0 & 53 & 0 & 59 \\
\hline 4 & 56 & 78 & 55 & 43 & 19 & 50 \\
\hline 5 & 45 & 62 & 52 & 47 & 17 & 34 \\
\hline 6 & 63 & 0 & 0 & 36 & 0 & 54 \\
\hline Média & 46,0 & 35,0 & 17,8 & 51,8 & 9,3 & 48,8 \\
\hline
\end{tabular}

de $36 \%$ a $69 \%$ nos grupos controles (Quadro 4). A resistência aos princípios ativos ABZ e IVM foi observada para ambos os gêneros de nematoides. A eficácia do ABZ e IVM para Haemonchus spp. foi igual à zero em $50 \%$ e $66,6 \%$ dos rebanhos, respectivamente. Outro fato que chama atenção é a baixa eficácia do ABZ para Trichostrongylus spp., inferior a $19 \%$.

Em ambos os biomas, com exceção de um único rebanho no qual a eficácia do MOX foi de $97 \%$, a eficácia dos anti-helmínticos foi inferior a 95\%. Não houve diferença estatística significativa entre a média do OPG e eficácia de albendazol e ivermectina entre os biomas Caatinga e Mata Atlântica.

\section{DISCUSSÃO}

Em rebanhos caprinos dos biomas Caatinga e Mata Atlântica, as infecções por NGIs foram moderadas e podem ser consideradas prejudiciais ao desempenho produtivo dos caprinos, quando se assume o ponto de corte para o OPG em infecções mistas superior a 800 (Ueno \& Goncalves 1998).

A resistência de NGIs ao ABZ foi observada em rebanhos de ambos os biomas. A baixa eficácia do anti-helmíntico ABZ foi condizente com os resultados de Lima et al. (2010b) que obtiveram de 30 a 75,5\% de redução do OPG em rebanhos caprinos da região do Cariri Paraibano, durante o período seco. Melo et al. (2009) encontraram a prevalência da resistência aos benzimidazóis de 87,5\% nas propriedade de caprinos, mesmo utilizando o dobro da dose de oxfendazol $(5 \mathrm{mg} / \mathrm{mL})$.

Vale salientar que a dose de ABZ recomendada para caprinos é $7,6 \mathrm{mg} / \mathrm{kg}$ de peso vivo (Silvestre et al. 2002), contudo não há no Brasil formulações comerciais disponíveis com a dose efetiva para essa espécie animal. Além disso, são escassos medicamentos anti-helmínticos com indicação na bula para o tratamento em caprinos. Portanto, é comumente usada a dose indicada para ovinos, de $5 \mathrm{mg} /$ $\mathrm{kg}$ ou até inferior a esta $(2,5 \mathrm{mg} / \mathrm{kg})$, como observada em produtos comercializados no mercado brasileiro. 0 uso de benzimidazóis em caprinos na dose indicada para ovinos é menos eficaz, uma vez que os caprinos biotransformam os benzimidazóis diferentemente dos ovinos, o que pode resultar em menores concentrações de metabólitos ativos no plasma e fluído gastrointestinal e no desenvolvimento da resistência aos anti-helmínticos (Hennessy et al. 1993).
A eficácia do ABZ (33,3\%) em rebanhos de Cansanção diverge do estudo de Ahid et al. (2007) na Zona da Mata em Alagoas, no qual encontrou-se, sete dias após a vermifugação, populações de nematoides sensíveis ao ABZ. Embora ambos os estudos tenham ocorrido em rebanhos na região nordeste, deve-se considerar que a taxa de desenvolvimento da resistência aos anti-helmínticos varia geograficamente (Leignel \& Humbert 2001, Saeed et al. 2010), na dependência de vários fatores incluindo o número de tratamentos, o uso repetido de um mesmo grupo químico (Silvestre et al. 2002) e a época do tratamento. Em relação a este último, Melo et al. (2009) salientaram que as dosificações anti-helmínticas no período seco podem acelerar o desenvolvimento da resistência na região semiárida do nordeste brasileiro, pois este período é caracterizado por uma população em refúgio pequena ou nula.

Populações de Haemonchus spp. resistentes ao LEV estão também distribuídas no município de Cansanção, enquanto os níveis de eficácia para o gênero Trichostrongylus foram superiores em relação aos outros compostos. Em rebanhos do Vale do Jiquiriçá não foi avaliado o LEV devido ao número insuficiente de animais. No semiárido paraibano, Rodrigues et al. (2007) verificaram eficácia superior a $94,2 \%$, qualificando os nematoides como moderadamente suscetíveis a ação do LEV, e posteriormente, Lima et al. (2010b) registraram menor eficácia, de 79\% no período seco e $69 \%$ no período chuvoso. Nesse bioma Caatinga, como em outros do Nordeste brasileiro os imidozotiazóis vem sendo pouco empregados na desparasitação dos animais (Melo et al. 2009, Coelho et al. 2010).

A eficácia reduzida para a IVM foi encontrada em todos os rebanhos. Diferentemente, em Mossoró, RN, o percentual de população de helmintos resistentes a IVM foi de $43,3 \%$ em 30 propriedades estudadas, o que pode ser relacionado ao menor uso do medicamento, uma vez que $33,3 \%$ dos rebanhos caprinos eram tratados com IVM, enquanto $63,3 \%$ com benzimidazóis (Coelho et al. 2010 ).

Uma das possíveis justificativas para os resultados do presente estudo no bioma caatinga é o fato de que a maioria dos animais era frequentemente tratado com IVM, conforme informações obtidas dos produtores. Além disso, a subdosagem pelo emprego de doses recomendadas para ovinos foi evidentemente praticada nestes rebanhos, uma vez que poucos registros de medicamentos anti-helmínticos discrimina o caprino (Rinaldi et al. 2012). A comparação da exposição à endectocidas em caprinos e ovinos mostrou que existem particularidades na cinética, e esta diferença é devido a menor biodisponibilidade em caprinos, por maior capacidade de biotransformação (Lespine et al. 2012). Porém, quando a dosagem de ivermectina foi ajustada para $0,3 \mathrm{mg} / \mathrm{kg}$, via oral, em caprinos, constatou-se a ausência de resistência (Paraud et al. 2010).

Em apenas um dos rebanhos do bioma Caatinga tratados com MOX foram encontrados nematoides suscetíveis. Em Pernambuco, Lima, M. et al. (2010a) registraram que a MOX não atingiu o nível necessário indicativo de eficácia, que foi de $91 \%$ em rebanhos caprinos. No entanto, Rodrigues et al. (2007) descreveram que nas fêmeas caprinas tratadas com MOX por via oral, os percentuais de eficácia 
foram de $92,8,88,7$ e $89,8 \%$ aos 7, 14 e 21 dias após o início do tratamento, respectivamente, e nos machos de 92,6, 96,2 e $98,1 \%$.

As maiores eficácias encontradas neste estudo para a MOX, apesar de não totalmente efetivas, podem ser esclarecidas pelo menor uso deste grupo químico ou por seu perfil farmacocinético, mais lipofílica do que outros endectocidas e maior biodisponibilidade no organismo animal, o que explica a maior área sob a curva de concentração plasmática como descrito por Lespine et al. (2012). A redução da contagem de ovos nas fezes de caprinos tratados com IVM foi muito baixa. A IVM e MOX estão na mesma classe de anti-helmíntico, lactonas macrociclicas, mas a maior potência de MOX produz elevada eficácia contra parasitos resistentes a IVM em caprinos, embora a resistência seja uma característica da classe do medicamento e não do fármaco especificamente (Howell et al. 2008).

Os níveis de resistência de NGIs foram mais semelhantes entre os rebanhos mais próximos, como também observado por Calvete et al. (2012), onde foi encontrada auto correlação espacial positiva para resistência parasitária entre os rebanhos localizados dentro de $30 \mathrm{~km}$. Entre outros pontos a serem destacados, vale ressaltar o modelo de produção destas regiões, especialmente a semiárida, o qual é predominantemente extensivo ou "fundo de pasto". 0 pastoreio em pastagens comunais, como também o comércio e a permuta de animais entre os criadores locais, provavelmente favorece a circulação e estabelecimento de parasitos resistentes (Silvestre et al. 2002). No município de Mossoró, RN, a resistência de NGI ao ABZ concentrou-se na região oeste, enquanto para IVM nas regiões oeste e sudeste (Coelho et al. 2010). 0 sistema de manejo semelhante entre os produtores de uma mesma localidade, assim como o uso dos mesmos grupos de anti-helmínticos e práticas similares de frequência e via de administração dos fármacos pode ser uma razão para justificar estes achados.

Nos biomas estudados, os resultados do presente trabalho revelam a dificuldade da indicação de medicamentos anti-helmínticos para controle dos NGIs mais frequentes de caprinos, visto o desenvolvimento da resistência para as principais classes de anti-helmíntico. Este fato sinaliza que as práticas do uso contínuo de várias bases químicas, diversas vezes por ano, e a subdosagem têm proporcionado à seleção de NGIs resistentes (Kumsa \& Abebe 2009, Calvete et al. 2012). Associado a isso, a dosagem incorreta pela estimativa visual do peso e indisponibilidade de formulações comerciais para caprinos e a escolha do anti-helmíntico sem nenhum critério técnico contribuíram para o desenvolvimento da resistência de nematoides nestes rebanhos.

\section{CONCLUSÃO}

Diante do exposto, concluímos que populações dos gêneros Haemonchus e Trichostrongylus resistentes ao ABZ, IVM, LEV, MOX e CLOS foram encontrados em rebanhos caprinos dos biomas Caatinga e Mata Atlântica, o que sugere a ocorrência da resistência anti-helmíntica múltipla.

Agradecimentos.- À FAPESB pelo financiamento da pesquisa (Editais Apoio à Tecnologias Sociais e Ambientais - Pedido № 6988/2009 e PRO-
NEN - Pedido № 1494/2011), a CAPES pela concessão de bolsa de mestrado. Ao biólogo Osnaldo Timóteo de Lima, Presidente do Sindicato Rural de Cansanção e ao Instituto Federal de Educação, Ciência e Tecnologia Baiano, Campus Santa Inês, pela identificação dos produtores. Ao médico veterinário Ademilton Silva e a assistente de laboratório Gilda de Souza Santos pelo auxilio nas análises parasitológicas.

\section{REFERÊNCIAS}

Ahid S.M.M., Cavalcante M.D.A., Bezerra A.C.D.S., Soares H.S. \& Pereira R.H.M.A. 2007. Eficácia anti-helmíntica em rebanho caprino no Estado de Alagoas, Brasil. Acta Vet. Brasilica 1:56-59.

Bahia. Superintendência de Estudos Econômicos e Sociais da Bahia. Sistema de Informações Municipais. Disponível em <http://sim.sei.ba.gov. br/sim/informacoes_municipais.wsp> Acesso em 28 dez. 2013.

Calvete C., Calavia R., Ferrer L.M., Ramos J.J., Lacasta D. \& Uriarte J. 2012. Management and environmental factors related to benzimidazole resistance in sheep nematodes in Northeast Spain. Vet. Parasitol. 184:193203.

Coelho W.A.C., Ahid S.M.M., Vieira L.S., Fonseca Z.A.A.S. \& Silva I.P. 2010. Resistência anti-helmíntica em caprinos no município de Mossoró. Ciênc. Anim. Bras. 11(3):589-599.

Coles G.C., Bauer C., Borgsteede F.H.M., Gets T.R., Klei T.R., Taylor M.A. \& Waller P.J. 1992. World Association for the Advancement of Veterinary Parasitology (WAAVP) methods for the detection of anthelmintic resistance in nematodes of veterinary importance. Vet. Parasitol. 44:35-44.

Hennessy D.R., Sangster N.C., Steel J.W. \& Collinsi G.H. 1993. Comparative pharmacokinetic behaviour of albendazole in sheep and goats. Int. J. Parasitol. 23(3):321-325.

Howell S.B., Burke J.M., Miller J.E., Terrill T.H., Valencia E., Williams M.J., Williamson L.H., Zajac A.M. \& Kaplan R.M. 2008. Prevalence of anthelmintic resistance on sheep and goat farms in the southeastern United States. J. Am. Vet. Med. Assoc. 233(12):1913-1919.

Kumsa B. \& Abebe G. 2009. Multiple anthelmintic resistance on a goat farm in Hawassa (southern Ethiopia). Trop. Anim. Health Prod. 41:655-662.

Leignel V. \& Humbert J.F. 2001. Mitochondrial DNA variation in benzimidazole-resistant and -susceptible of the small rumninant parasite Teladorsagia circumcincta. J. Hered. 92(6):503-506.

Lespine A., Chartier C., Hoste H. \& Alvinerie M. 2012. Endectocides in goats: Pharmacology, efficacy and use conditions in the context of anthelmintics resistance. Small Rumin. Res. 103:10-17.

Lima M.M., Farias M.P.O., Romeiro E.T., Ferreira D.R.A., Alves L.C. \& Faustino M.A.G. 2010. Eficácia da moxidectina, ivermectina e albendazole contra helmintos gastrintestinais em propriedades de criação caprina e ovina no estado de Pernambuco. Ciênc. Anim. Bras. 11(1):94-100.

Lima W.C., Athayde A.C.R., Medeiros G.R., Lima D.A.S.D., Borburema J.B., Santos E.M., Vilela V.L.R. \& Azevedo S.S. 2010. Nematóides resistentes a alguns anti-helmínticos em rebanhos caprinos no Cariri Paraibano. Pesq. Vet. Bras. 30(1):1003-1009.

Melo A.C.F.L, Bevilaqua C.M.L. \& Reis I.F. 2009. Resistência aos anti-helmínticos benzimidazóis em nematóides gastrintestinais de pequenos ruminantes do semiárido nordestino brasileiro. Ciênc. Anim. Bras. 10(1):294-300.

Molento M.B., Niciura S.C.M. \& Chagas A.C.S. 2011. Protocolos básicos de laboratório para realização de metodologias fenotípicas e genotípicas, 117-143. In: Ibid. (Eds), Manual Prático: metodologias de diagnóstico da resistência e de detecção de substancias ativas em parasitas de ruminantes. Embrapa Informação Tecnológica, Brasília.

Paraud C., Pors I., Rehby L. \& Chartier C. 2010. Absence of ivermectin resistance in a survey on dairy goat nematodes in France. Parasitol. Res. 106:1475-1479.

Prichard R.K., Hall C.A., Kelly J.D., Martin I.C.A. \& Donald A.D. 1980. The problem of anthelmintic resistance in nematodes. Aust. Vet. J. 56:239250. 
Rinaldi L.E. \& Cringoli G. 2012. Parasitological and pathophysiological methods for selective application of anthelmintic treatments in goats. Small Rumin. Res. 10:18-22.

Rodrigues A.B., Athayde A.C.R., Rodrigues O.G., Silva W.W. \& Faria E.B. 2007. Sensibilidade dos nematóides gastrintestinais de caprinos a anti-helmínticos na mesorregião do Sertão Paraibano. Pesq. Vet. Bras. 27(4):162-166.

Saeed M., Iqbal Z., Jabbar A., Masood S., Babar W., Saddiqi H.A, Yaseen M., Sarwar M. \& Arshad M. 2010. Multiple anthelmintic resistance and the possible contributory factors in Beetal goats in an irrigated area (Pakistan). Res. Vet. Sci. 88.297-272.

Silvestre A., Leignel V., Berrag B., Gasnier N., Humbert J.F., Chartier C. \& Cabaret J. 2002. Sheep and goat nematode resistance to anthelmintics: pro and cons among breeding management factors. Vet. Res. 33:465480.

Ueno H. \& Gonçalves P.C. 1998. Manual para Diagnóstico das Helmintoses de Ruminantes. 4th ed. Japan International Cooperation, Tokio. $143 p$.

Vieira L.S. \& Cavalcante A.C.R. 1999. Resistência antihelmíntica em rebanhos caprinos no Estado do Ceará. Pesq. Vet. Bras. 19(3/4):99-103.

Voltolini T.V., Moraes S.A. de, Araujo G.G.L. de \& Santos R.M. 2011. Principais modelos produtivos na criação de caprinos e ovinos, p.219-232. In: Voltolini T.V. (Ed.). Produção de caprinos e ovinos no Semiárido. Embrapa Semiárido, Petrolina, PE.

Waruiru R.M., Kogi J.K., Weda E.H. \& Ngotho J.W. 1998. Multiple anthelmintic resistance on a goat farm in Kenya. Vet. Parasitol. 75:191-197. 